\title{
Correlation equalities and upper bounds for the transverse Ising model
}

\author{
F C Sá Barreto and A L Mota \\ Departamento de Ciências Naturais, Universidade Federal de São João del Rei, C.P. \\ 110, CEP 36301-160, São João del Rei, Brazil \\ E-mail: fcsabarreto@gmail.com, motaal@ufsj.edu.br
}

\begin{abstract}
Starting from an exact formal identity for the two-state transverse Ising model and using correlation inequalities rigorous upper bounds for the critical temperature and the critical transverse field are obtained which improve effective results.

PACS numbers: 75.30.kz,75.10.Jm,64.60.A-
\end{abstract}




\section{Introduction}

The transverse Ising model (TIM) is described by a two-state Ising Hamiltonian with a term representing a field transverse to the spins,

$$
H=-\sum_{i j} J_{i j} S_{i}^{z} S_{j}^{z}-\Omega \sum_{i} S_{i}^{x}
$$

where $J_{i j}>0, \Omega$ is the transverse field, $S_{i}^{z}$ and $S_{i}^{x}$ are Pauli spin- $\frac{1}{2}$ operators and the first sum is over the nearest neighbors spins on the lattice. The model has been firstly applied to describe the phase transitions and the properties of hydrogen bonded ferroelectrics [1, 2] and magnetic ordered materials [3]. This model in one dimension has no phase transition at finite temperatures; however, at zero temperature it is ordered up to the critical value of the transverse field. The model has been solved exactly in one dimension [4, 5, 6]. In high dimensions there are approximations for low-temperatures or high-temperatures regions [7, 8]. All other calculations are based on mean field type approximations. An effective field theory has been presented which improve over mean field results [9]. Since then many results have been obtained based on the effective field theory. More recently the model has been used to study the phase diagrams of nanowires systems [10] and magnetization of nanoparticles [11] . The objective of this paper is to present rigorous upper bounds for the critical couplings. We will apply the results for the $\mathrm{d}=2$ square lattice and the $\mathrm{d}=3$ cubic lattice.

\section{Generalized Callen's identity for the transverse Ising model}

In this section, we describe the methodology used by Sá Barreto et al [9] to derive an identity for the two-spin correlation function of the transverse Ising model. The procedure used in this deduction was presented in reference [9] to obtain an exact relation for the order parameter $\left\langle S_{i}^{z}\right\rangle$ which generalizes Callen's identity [12]. The longitudinal two-spin correlation function $\left.<S_{l}^{z} S_{i}^{z}\right\rangle$ can be calculated from

$$
<S_{l}^{z} S_{i}^{z}>=\frac{\operatorname{Tr}\left(e^{-\beta H} S_{l}^{z} S_{i}^{z}\right)}{\operatorname{Tr}\left(e^{-\beta H}\right)}
$$

where $\mathrm{H}$ is given by (1). The Hamiltonian can be separated into two parts, $H=H_{i}+H^{\prime}$, where $H_{i}$ includes all parts associated with site $\mathrm{i}$ and $H^{\prime}$ represents the rest of the Hamiltonian. A direct calculation leads to

$$
\left.<S_{l}^{z} S_{i}^{z}\right\rangle=\left\langle S_{l}^{z} \frac{\operatorname{Tr}_{(i)} S_{i}^{z} e^{-\beta H_{i}}}{\operatorname{Tr}_{(i)} e^{-\beta H_{i}}}\right\rangle-\left\langle S_{l}^{z}\left[\frac{\operatorname{Tr}_{(i)} S_{i}^{z} e^{-\beta H_{i}}}{\operatorname{Tr}_{(i)} S_{i}^{z} e^{-\beta H_{i}}}-S_{i}^{z}\right] \Delta\right\rangle
$$

where $\operatorname{Tr}_{(i)}$ represents the partial trace with respect to site $\mathrm{i}$ and $\Delta=1-$ $e^{-\beta H_{i}} e^{-\beta H^{\prime}} e^{\beta\left(H_{i}+H^{\prime}\right)}$. Equation (3) is an exact relation. However, it is difficult to be used. Therefore, we will make an approximation based on the following decoupling,

$$
\left\langle S_{l}^{z}\left[\frac{\operatorname{Tr}_{(i)} S_{i}^{z} e^{-\beta H_{i}}}{\operatorname{Tr}_{(i)} e^{-\beta H_{i}}}-S_{i}^{z}\right] \Delta\right\rangle \approx\left\langle S_{l}^{z}\left[\frac{\operatorname{Tr}_{(i)} S_{i}^{z} e^{-\beta H_{i}}}{\operatorname{Tr}_{(i)} e^{-\beta H_{i}}}-S_{i}^{z}\right]\right\rangle<\Delta>
$$


Inserting (44) into (3) and using the fact that $<\Delta>\leq 1$, we obtain,

$$
<S_{l}^{z} S_{i}^{z}>\leq\left\langle S_{l}^{z} \frac{\operatorname{Tr}_{(i)} S_{i}^{z} e^{-\beta H_{i}}}{\operatorname{Tr}_{(i)} e^{-\beta H_{i}}}\right\rangle
$$

By expanding $\Delta$ we see that the approximation is correct to the order of $\beta^{2}$. Moreover, it is consistent with the application of the correlation inequalities that will be used later to obtain the upper bounds for the critical couplings. In the next steps we will keep only the $=\operatorname{sign}$ of $(\sqrt[5]{1})$.

Let us write $H_{i}=-E_{i} S_{i}^{z}-\Omega S_{i}^{x}$, where $E_{i}=\sum_{j} J_{i j} S_{j}^{z}$. Diagonalizing and taking the partial trace over $\mathrm{i}$, we get for the longitudinal spin correlation function, $\left\langle\sigma_{l}^{z} \sigma_{i}^{z}\right\rangle$, where $\sigma_{i}=2 S_{i}$,

$$
\begin{aligned}
& <\sigma_{l}^{z} \sigma_{i}^{z}>=\left\langle\sigma_{l}^{z} \frac{\sum_{j} J_{i j} \sigma_{j}^{z}}{\sqrt{(2 \Omega)^{2}+\left(\sum_{j} J_{i j} \sigma_{j}^{z}\right)^{2}}} \times\right. \\
& \left.\tanh \left(\beta \sqrt{(2 \Omega)^{2}+\left(\sum_{j} J_{i j} \sigma_{j}^{z}\right)^{2}}\right)\right\rangle .
\end{aligned}
$$

Introducing the exponential operator $e^{(a D)} \cdot f(x)=f(x+a), D=\frac{\partial}{\partial x}$, we obtain,

$$
\begin{aligned}
& <\sigma_{l}^{z} \sigma_{i}^{z}>=\left.\left\langle\sigma_{l}^{z} e^{\sum_{j}\left(J_{i j} \sigma_{j}^{z}\right) D}\right\rangle \cdot f(x)\right|_{x=0} \\
& =\left.\left\langle\sigma_{l}^{z} \sigma_{j}^{z} \prod_{j} e^{\left(J_{i j} \sigma_{j}^{z}\right) D}\right\rangle \cdot f(x)\right|_{x=0}
\end{aligned}
$$

where $\mathrm{f}(\mathrm{x})$ is given by

$$
f(x)=\frac{x}{\sqrt{(2 \Omega)^{2}+x^{2}}} \tanh (\beta) \sqrt{(2 \Omega)^{2}+x^{2}}
$$

Note that $f(x)=-f(-x)$.

Expanding the exponential in (77) and considering that $\left(\sigma_{i}^{z}\right)^{2}=1$, we obtain,

$$
\left\langle\sigma_{l}^{z} \sigma_{i}^{z}\right\rangle=\left.\left\langle\sigma_{l}^{z} \prod_{j}\left[\cosh \left(J_{i j} D\right)+\sigma_{i}^{z} \sinh \left(J_{i j} D\right)\right]\right\rangle \quad \cdot f(x)\right|_{x=0}
$$

By a similar procedure the transverse two-spin correlation function $\left\langle\sigma_{l}^{x} \sigma_{i}^{x}\right\rangle$ is obtained,

$$
\left.<\sigma_{l}^{x} \sigma_{i}^{x}\right\rangle=\left.\left\langle\sigma_{l}^{x} \prod_{j}\left[\cosh \left(J_{i j} D\right)+\sigma_{i}^{z} \sinh \left(J_{i j} D\right)\right]\right\rangle \cdot g(x)\right|_{x=0}
$$

where $\mathrm{g}(\mathrm{x})$ is

$$
g(x)=\frac{2 \Omega}{\sqrt{(2 \Omega)^{2}+x^{2}}} \tanh (\beta) \sqrt{(2 \Omega)^{2}+x^{2}}=g(-x)
$$

The expectation value of $\sigma_{i}^{x}$ is given by,

$$
<\sigma_{i}^{x}>=\left.\left\langle\prod_{j}\left[\cosh \left(J_{i j} D\right)+\sigma_{i}^{z} \sinh \left(J_{i j} D\right)\right]\right\rangle \cdot g(x)\right|_{x=0}
$$




\section{Application to $d=2$ square lattice and $d=3$ cubic lattice.}

\section{1. $d=2$ lattice}

Considering the four neighbours of $i$ in (9), expanding the product, applying the exponential operators appearing in the powers of $\cosh \left(J_{i j} D\right)$ and $\sinh \left(J_{i j} D\right)$ in $\mathrm{f}(\mathrm{x})$, we obtain,

$$
<\sigma_{l}^{z} \sigma_{i}^{z}>=A_{2} \sum_{j}<\sigma_{l}^{z} \sigma_{j}^{z}>+B_{2} \sum_{j<k<m}<\sigma_{l}^{z} \sigma_{j}^{z} \sigma_{k}^{z} \sigma_{m}^{z}>
$$

where

$$
\begin{aligned}
& A_{2}=\frac{1}{8}[f(4 J)+2 f(2 J)]>0 \\
& B_{2}=\frac{1}{8}[f(4 J)-2 f(2 J)]<0
\end{aligned}
$$

and $j, k$ and $m$ are neighbours of $i$ and $f(.$.$) is given by (8)$.

\section{2. $d=3$ lattice.}

After a similar calculation we obtain for the cubic lattice,

$$
\begin{aligned}
& <\sigma_{l}^{z} \sigma_{i}^{z}>=A_{3} \sum_{j}<\sigma_{l}^{z} \sigma_{j}^{z}>+B_{3} \sum_{j<k<m}<\sigma_{l}^{z} \sigma_{j}^{z} \sigma_{k}^{z} \sigma_{m}^{z}> \\
& +C_{3} \sum_{j<k<m<n<p}<\sigma_{l}^{z} \sigma_{j}^{z} \sigma_{k}^{z} \sigma_{m}^{z} \sigma_{n}^{z} \sigma_{p}^{z}>
\end{aligned}
$$

where,

$$
\begin{aligned}
& A_{3}=\frac{1}{2^{5}}[f(6 J)+4 f(4 J)+5 f(2 J)]>0 \\
& B_{3}=\frac{1}{2^{5}}[f(6 J)-3 f(2 J)]<0 \\
& C_{3}=\frac{1}{2^{5}}[f(6 J)-4 f(4 J)+5 f(2 J)]>0
\end{aligned}
$$

and $j, k, m, n$ and $p$ are neighbours of $i$ and $f(.$.$) is given by (8).$

\section{Exponential decay of the two-point functions and the upper bounds.}

Upper bounds for the critical temperature $T_{c}$ for Ising and multi-component spin systems have been obtained by showing (for $T>T_{c}$ ) the exponential decay of the two-point function [13, 14, 15. The procedure to obtain these upper bounds for the critical couplings of the tranverse Ising model is the following: we start from a two-point correlation function equations (13) and (15) and we make use of Griffiths inequalities (Griffiths I, II) [16, 18, 19] and Newman and Lebowitz inequalities [17, 19] . A proof of Griffiths inequalities has been given for the XY model with no external field [16]. Extensions of Griffiths-Kelly-Sherman inequalities to quantal systems, under external 
fields, both longitudinal and transverse, have been proved [18, 19]. The physical reason why the Griffiths and similar inequalities are valid for the quantal XY-type Hamiltonian is that the off-diagonal interaction, namely, $H_{1}(x)=\sum_{A} J_{A}^{x} \sigma_{A}^{x},\left(J_{A}^{x} \leq 0\right)$, produce the decrease of the ferromagnetic correlation among the $\sigma_{j}^{z}$-spins, but it is not sufficient big to create a cooperative effect to induce an antiferromagnetic correlation. In other words, one can say that $H_{1}(x)$ is a dynamical random force acting on $\mathrm{Z}-\mathrm{z}$ correlations [18]. We establish the inequality for the two-point function $\left\langle\sigma_{l}^{z} \sigma_{i}^{z}\right\rangle$,

$$
<\sigma_{l}^{z} \sigma_{i}^{z}>\leq \sum_{j} a_{j}<\sigma_{l}^{z} \sigma_{j}^{z}>, 0 \leq a_{j} \leq 1
$$

which when iterated [14] implies exponential decay for $T>T_{c}$.

\subsection{Upper bounds for $d=2$.}

From Eq.(13), using Griffiths II $\left(<\sigma_{l}^{z} \sigma_{j}^{z} \sigma_{k}^{z} \sigma_{m}^{z}>\geq<\sigma_{l}^{z} \sigma_{j}^{z}><\sigma_{k}^{z} \sigma_{m}^{z}>\right)$ in the second term and considering $B_{2}<0$, we get,

$$
<\sigma_{l}^{z} \sigma_{i}^{z}>\leq \sum_{j} a_{j}<\sigma_{l}^{z} \sigma_{j}^{z}>
$$

where $j \neq k$ are neighbours of $i$, and

$$
a_{j}=A_{2}-\left|B_{2}\right|<\sigma_{j}^{z} \sigma_{k}^{z}>_{1 d}
$$

\subsection{Upper bounds for $d=3$.}

From Eq.(15), using Griffiths II in the second term $B_{3}<0$, Newman's inequality $\left(<\sigma_{i}^{z} F>\leq \sum_{j}<\sigma_{i}^{z} \sigma_{j}^{z}><d F / d \sigma_{j}^{z}>, \mathrm{F}\right.$ are polynomials with positive coefficients) combined with Griffiths I $\left(<\sigma_{A}^{z}>\leq 1\right)$ on the third term $C_{3}>0$, we get,

$$
<\sigma_{l}^{z} \sigma_{i}^{z}>\leq \sum_{j} a_{j}<\sigma_{l}^{z} \sigma_{j}^{z}>
$$

where $j \neq k$ are neighbours of $i$, and

$$
a_{j}=A_{3}-\left|B_{3}\right|<\sigma_{j}^{z} \sigma_{k}^{z}>_{1 d}+5 C_{3}
$$

\subsection{Numerical Results}

The two-spin correlation functions appearing in (19) and (21) $<\sigma_{j}^{z} \sigma_{k}^{z}>_{1 d}$ is the onedimensional model two-spin correlation function separated by a distance of two lattices sites. For the one-dimensional transverse Ising model the exact value of this function at the critical value $\Omega_{c}=J$ is [4]:

$$
<\sigma_{j}^{z} \sigma_{k}^{z}>_{1 d}=\frac{1}{4}\left(\frac{2}{\pi}\right)^{2} 8 \frac{H^{4}(2)}{H(4)}
$$

where $H(n)=1^{n-1} 2^{n-2} \ldots(n-1)$. For the one-dimensional Ising model the exact value of the spin correlation function separated by a distance of two lattices sites is:

$$
<\sigma_{j}^{z} \sigma_{k}^{z}>_{1 d}=\tanh ^{2} \beta J
$$


In the following, we will use result (22) in (19) and (21) to calculate the upper bound for $\Omega_{c}$ at $T_{c}=0$ and result (23) in (19) and (21) to calculate the upper bound for $T_{c}$ at $\Omega=0$. Evaluating numerically the value of $\mathrm{T}$ such that $\sum_{j} a_{j} \leq 1, a_{j}>0$, we obtain, by sufficient condition (see Eq.(17)), the upper bounds for $T_{c}$ as a function of $\Omega$, shown in figure 1, together with the curve for the mean field results. We use (23) for the one-dimensional two-spin correlation function in obtaining figure 1. This curve represents the rigorous upper bounds for $T_{c}, \Omega_{c}$. In particular, the mean field values are $\Omega_{c}^{M F A}=z J / 2$ and $T_{c}^{M F A}(0)=z J / k_{B}$. The values for $\Omega_{c}$ and $T_{c}(0)$, which are the rigorous upper bounds for $d=2, z=4$ and $d=3, z=6$, obtained in the present calculation are: (a) $\mathrm{d}=2, \mathrm{z}=4 ; \frac{k_{B} T_{c}}{J}=3.014$ and $\Omega_{c}=1.3755 J$, (b) $\mathrm{d}=3, \mathrm{z}=6$; $\frac{k_{B} T_{c}}{J}=5.423$ and $\Omega_{c}=2.4466 J$.

In table 1, we compare the results obtained by the effective field calculation (EFT) [9], the high temperature expansion (HTE) [7, 8] and the present results for $\Omega_{c}$.

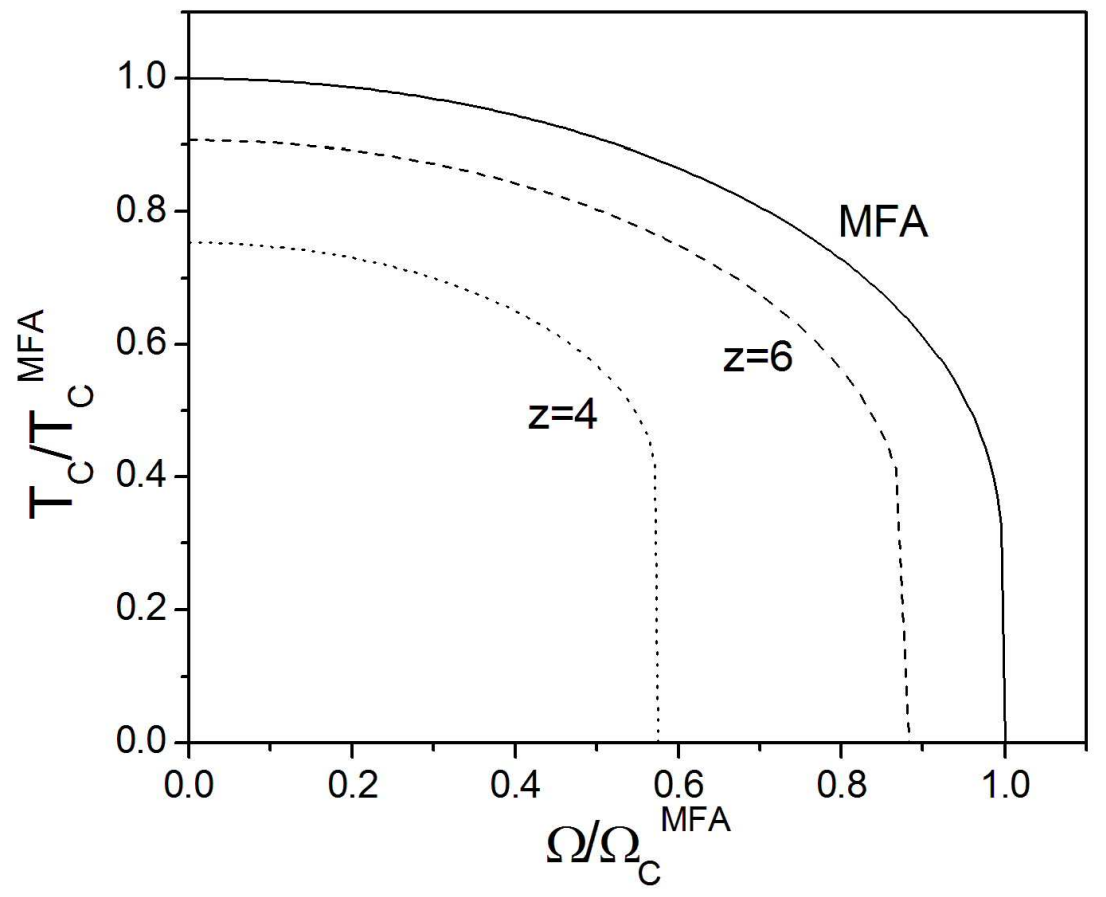

Figure 1. Phase diagram of the TIM model for $z=4$ and $z=6$. Solid line is the mean field approximation result. Results for our approach evaluated by using the spin correlation function separated by two sites, Eq.(23) are given by the dotted $(z=4)$ and dashed $(z=6)$ lines. 
Table 1. Estimatives for $\Omega_{c} / \Omega_{c} M F A$ for $\mathrm{d}=2$ and $\mathrm{d}=3$.

\begin{tabular}{ccc}
\hline & $d=2, z=4$ & $d=3, z=6$ \\
\hline MFA & 1 & 1 \\
EFT & 0.688 & 0.784 \\
HTE & 0.770 & 0.860 \\
Present work & 0.643 & 0.813 \\
\hline
\end{tabular}

\section{Concluding remarks}

In this paper we have obtained rigorous upper bounds for the critical couplings of the transverse Ising model. The procedure was based on an approximation for an exact identity for the two-spin correlation functions and on rigorous inequalities for the spin correlation functions. The approximated relation for the two-spin correlation function, Eq.(5), used in this procedure, is consistent with the rigorous inequalities, Eq.(17), since both act in the same direction of the inequalities. The upper bounds were applied for two- and three- dimensional models.

\section{Acknowledgements}

FCSB is grateful for the financial support of CAPES/Brazil which made possible his visit to the UFSJ/Brasil. ALM acknowledges financial support from CNPq/Brazil and FAPEMIG/Brazil.

\section{References}

[1] de Gennes P G, Collective motions of hydrogen bonds, 1963 Solid St. Comm. 1132

[2] Blinc R and Zeks B, Dynamics of order-disorder-type ferroelectrics and anti-ferroelectrics, 1972 Adv. in Phys. 91693

[3] Wang Y L and Cooper B, Collective Excitations and Magnetic Ordering in Materials with Singlet Crystal-Field Ground State,1968 Phys. Rev. 172539

[4] Pfeuty P , The One-Dimensional Ising Model with a Transverse Field, 1970 Ann. Phys. 5779

[5] Katsura S, Statistical Mechanics of the Anisotropic Linear Heisenberg Model, 1968 Phys. Rev. 1271508

[6] Suzuki M, Equivalence of the two-dimensional Ising model to the ground state of the linear XYmodel, 1971 Phys. Lett. 34 A 94

[7] Elliott R J and Wood C, The Ising model with a transverse field. I. High temperature expansion,1971 J. Phys. C 42359

[8] Pfeuty P and Elliott R J, The Ising model with a transverse field. II. Ground state properties,1971 J. Phys C 42370

[9] Sá Barreto F C , Fittipaldi I P and Zeks B, New Effective Field Theory for the Transverse Ising Model, 1981 Ferroelectrics 391103

[10] Kaneyoshi T, Phase diagrams of a transverse Ising nanowire, 2010 J.Magn. Magn. Mater. 322 3014

[11] Kaneyoshi T, Magnetizations of a nanoparticle described by the transverse Ising model, 2009 J.Magn. Magn. Mater. 3213430 
[12] Callen H B, A note on Green functions and the Ising model,1963 Phys.Lett. 4161

[13] Fisher M, Critical Temperatures of Anisotropic Ising Lattices. II. General Upper Bounds, 1967 Phys.Rev. 162480

[14] Simon B, Correlation Inequalities and the Decay of Correlations in Ferromagnets, 1980 Commun.Math Phys. 77111

[15] Brydges D, Frolich J and Spencer T, The random walk representation of classical spin systems and correlation inequalities, 1982 Commun.Math.Phys. 83123

[16] Gallavotti G, A proof of the griffiths inequalities for the XY model, 1971 Studies Appl. Math 189

[17] Newman C, Gaussian Correlation Inequalities for Ferromagnets,1975 Zeitschriftfur WahrscheinlichkeitsTheorie 3375

[18] Suzuki M, Correlation inequalities and phase transition in the generalized X-Y model, 1973 J. Math. Phys. 12837.

[19] Contucci P and Lebowitz J L, Correlation inequalities for the quantum spin systems with quenched centered disorder, 2010 J. Math. Phys. 51023302 